\title{
An Experimental Study of Heat Transfer Enhancement in the Circular Channel with Almond Shape Dimples
}

\author{
Pooja Patil ${ }^{1}$, Prof.Padmakar Deshmukh ${ }^{2}$ \\ ${ }^{I}$ (Mechanical,JSPM'sRajrshi Shahu College of Engg,Pune /Pune University,India) \\ ${ }^{2}$ (Mechanical,JSPM'sRajrshi Shahu College of Engg,Pune /Pune University,India)
}

\begin{abstract}
Heat transfer coefficients were measured in a circular tube with dimpled surface. The almond type dimples were fabricated, the tube diameter (D) $19 \mathrm{~mm}$ and dimple depth $3 \mathrm{~mm}$ ratio was kept constant, while holding diameter $10 \mathrm{~mm}$ of dimple is required length $21.5 \mathrm{~mm}$ because of the elongated shape. The Reynolds number based on the channel hydraulic diameter was varied from 25000to 95000. An experimental study was conducted for the staggered configuration of the dimple and compare with the base line results of plain tube. In addition to this numerical investigation was carried out for similar experimental conditions with addition to align array of dimple. It was found that staggered array of dimple in circular tube has $66 \%$ greater thermal performance factor than align dimple configuration. Heat transfer results are compared with the base line with plain tube. Heat transfer measurement results showed that high heat transfer was induced downstream of the dimples due to flow reattachment. As the Reynolds increased, the overall heat transfer coefficients also increased. For the staggered dimple arrangement, the heat transfer coefficients and the thermal performance factors were higher for the same mass flow rate.
\end{abstract}

Keywords: almond dimple, enhancement, mass flow ,Renolds number, staggered, thermal performance factor

\section{Introduction}

One method to increase the convective heat transfer is to manage the growth of the thermal boundary layer. The thermal boundary layer can be made thinner or partially broken by flow disturbance. Disruption of the laminar sub layer in the turbulent boundary layer is one of a particularly important heat transfer mechanism for augmenting heat transfer. The disruption can be obtained by using rough wall surfaces. In recent years, the concept of using an indented (dimpled) surface instead of protruding devices has gained attention because of the combination of high heat transfer enhancement and a lower pressure loss penalty.

Johann Turnow et al. Studied Vortex structures and heat transfer enhancement mechanism of turbulent flow over a staggered array of dimples in a narrow channel. It was found that the dimple package with a depth $\mathrm{h}$ to diameter $\mathrm{D}$ ratio of $\mathrm{h} / \mathrm{D}=0.26$ provides the maximum thermo-hydraulic performance. The heat transfer rate could be enhanced up to $201 \%$ compared to a smooth channel [1]. Yu Rao et al. investigate the effects of dimple depth on the pressure loss and heat transfer characteristics in a pin fin-dimple channel, where dimples are located on the end wall transversely between the pin fins. The study showed that, compared to the baseline pin fin channel, the pin fin-dimple channels have further improved convective heat transfer performance by up to $19.0 \%$, and the pin fin-dimple channel with shallower dimples shows relatively lower friction factors by up to $17.6 \%$ over the Reynolds number range 8200 to 50,500[2]. C. Bi et al. studied convective cooling heat transfer in mini-channels with dimples, cylindrical grooves and low fins. The results show that the dimple surface presents the highest performance of heat transfer enhancement [3]. Chyu et al. studied the enhancement of surface heat transfer in a channel using two different concavities- hemispheric and tear drop. Concavities serve as vortex generators to promote turbulent mixing in the bulk flow to enhance the heat transfer at $\mathrm{ReH}=10,000$ to $50,000, \mathrm{H} / \mathrm{d}$ of $0.5,1.5,3.0$ and $\delta / \mathrm{d}=0.575$. Heat transfer enhancement was 2.5 times higher than smooth channel values and with very low pressure losses that were almost half that caused by conventional ribs turbulators [13].S.A. Isaev studied Influence of the Reynolds number and the spherical dimple depth on turbulent heat transfer and hydraulic loss in a narrow channel. Detailed information gained from the presented computations can be used to get a deep insight into flow physics over dimpled surfaces and as a benchmark for validation of numerical and experimental methods [4]. Jonghyeok Lee wt et al. developed Correlations and shape optimization in a channel with aligned dimples and protrusions. The friction factor and Nusselt number in a plate heat exchanger with dimples and protrusions were investigated according to geometric and operating conditions[5].Somin Shin et al. were measured Heat transfer coefficients in a channel with one side dimpled surface. The sphere type dimples were fabricated for the different diameter channel heights. The Reynolds number based on the channel hydraulic diameter was varied from 30000 to 50000 . Heat transfer measurement results showed that high heat transfer was induced downstream of the dimples due to flow reattachment. Due to the flow recirculation on the upstream side in the dimple, the heat transfer coefficient was very low. As the Reynolds increased, the overall heat transfer coefficients also increased. With the same dimple arrangement, the 
heat transfer coefficients and the thermal performance factors were higher for the lower channel height. As the distance between the dimples became smaller, the overall heat transfer coefficient and the thermal performance factors increased [6]. Yu Rao et al. An experimental and numerical study was conducted to investigate the flow friction and heat transfer performance in rectangular channels with staggered arrays of pin fin-dimple hybrid structures and pin fins in the Reynolds number range of 8200-54,000.Experimenta and numerical compared data showed that, compared with the pin fin channel, the pin fin-dimple channel has further improved convective heat transfer performance by about $8.0 \%$ and whereas lowered flow friction by more than $18.0 \%$.The computations showed that the dimples increase the near-wall turbulent mixing level by producing strong vortex flows, and therefore enhance the convective heat transfer in the channel. On the other hand, the dimples enlarge the minimum cross section area transversely between the pin fins, and therefore the pressure loss in the flow can be reduced in the pin fin-dimple channels.[7] Yu Chen studied a systematic numerical investigation of heat transfer in turbulent channel flow over dimpled surface. The tow dimple configurations were studied that is symmetric and asymmetric, for $\mathrm{h} / \mathrm{d}$. It was found that the heat transfer enhancement would be $15 \%$ for asymmetric $\mathrm{h} / \mathrm{d}$ with minimum loss of pressure as compare to symmetric dimples [8]. Nopparat Katkhaw et al. studied the Heat transfer behavior of flat plate having $45^{0}$ ellipsoidal dimpled surfaces. 10 types of dimple arrangements and dimple intervals are studied. For the staggered arrangement of dimple it was found that $15.8 \%$ heat transfer was improved as compare to smooth channel [9]. M. Siddique et al. made a review article for the recent advances in heat transfer enhancement [10].

From the published literature, it can be seen that there is a lot of experimental and numerical data available on the use of dimpled surfaces. However, there is a scarcity of literature available for dimple surface in the Turbulent flow regime inside circular tube. There is a need to gain a better insight in to the nature of Turbulent flows over dimpled surface. An investigation to see the effects of different array geometries is also needed. This study is carried out to see whether almond shape dimples can enhance heat transfer and thermal performance for turbulent airflows in a circular channel for two different array geometries using Computational fluid dynamics. And experimental work is carried out for the geometry which gives better performance.

\section{Experimental Set up}

The apparatus consists of a centrifugal blower unit fitted with a circular tube, which is connected to the test tube located in horizontal orientation. Flexi glass heater encloses the test section to a whole length of $1000 \mathrm{~mm}$. Input to heater is given through rheostat. Four thermo couples $T_{1}, T_{2}, T_{3}$ and $T_{4}$ at a equal distance of $15 \mathrm{~cm}$ from the origin of the heating zone are embedded on the walls of the tube and one thermocouple is placed in the air stream at the exit $\left(\mathrm{T}_{5}\right)$ of the test section to measure the temperature of flowing air. The digital device multimeter is used to display the temperature measured by thermocouple at various position. The temperature measured by instrument is in ${ }^{0} \mathrm{C}$. The test tube of $3 \mathrm{~mm}$ thickness is used for experimentation. A U tube manometer measures the pressure drop across the test section filled with water. The pipe system consists of a valve, which controls the airflow rate through it and an orifice meter to find the volume flow rate of air through the system. The diameter of the orifice is $.0125 \mathrm{~m}$ and coefficient of discharge is 0.61 . The two pressure tapings of the orifice meter are connected to a water U-tube manometer to indicate the pressure difference between them. Display unit is a digital miltimeter used to indicate temperature indicator. The circuit is designed for a load voltage of 0-100 V; with a maximum current of 10 A. Difference in the levels of manometer fluid represents the variations in the flow rate of air. The velocity of airflow in the tube is measured with the help of orifice plate and the water manometer fitted on board.

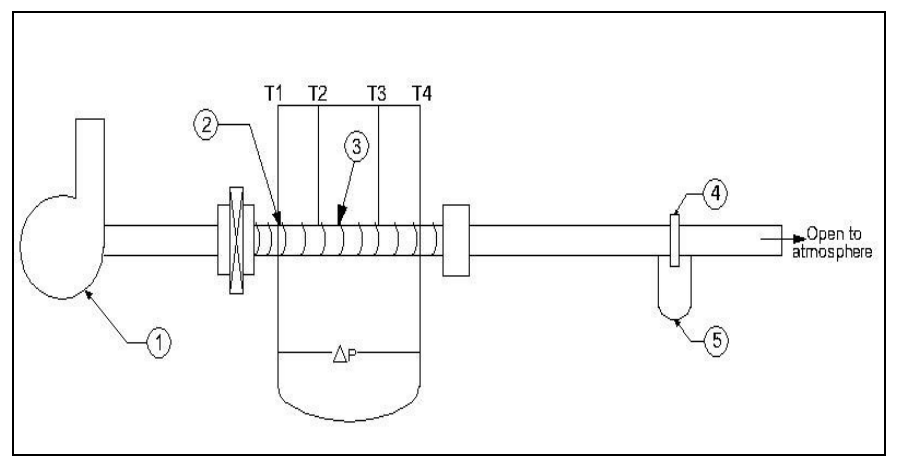

Fig.1: Schematic diagram of the Experimental Set-up

1. Blower, 2.Test Tube, 3.Heating Coil, 4.Orifice, 5.U tube Manometer, 6. $T_{1}$ to $T_{4}$ Thermocouples, $\Delta P$ : inlet outlet pressure difference of test tube. 


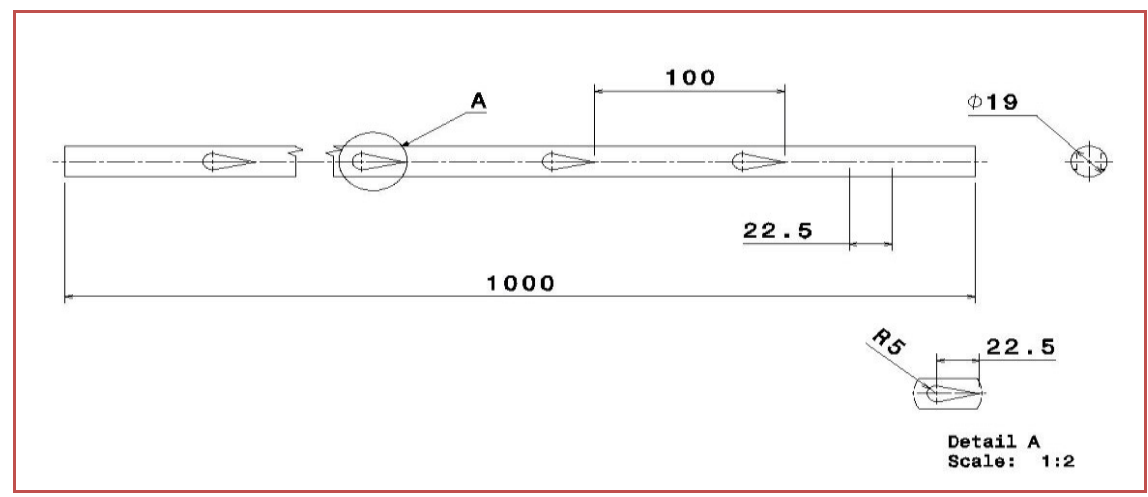

Fig.2: Geometrical Configuration of Test tube

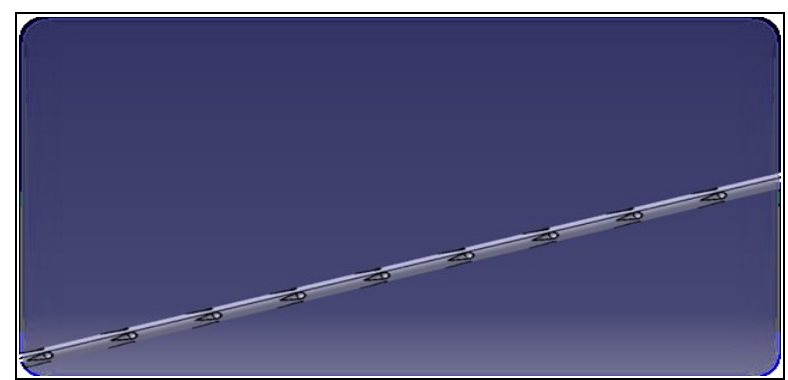

Fig.3: Tube with almond shape dimples

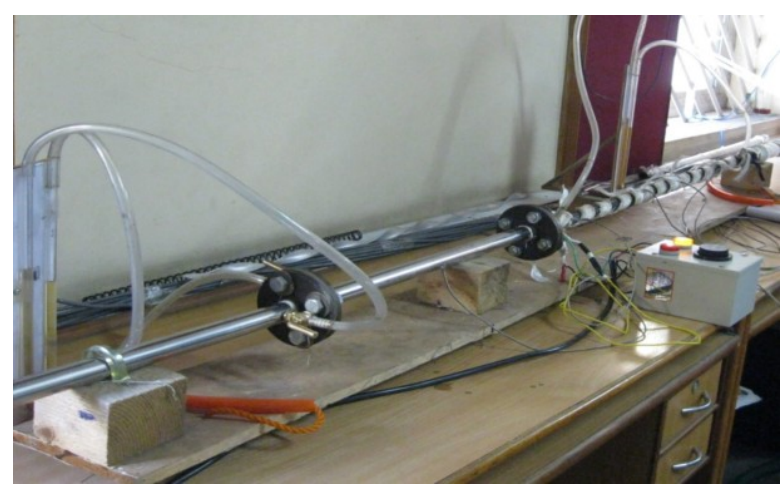

Fig.4: Experimental set up

\section{Manufacturing of the Test Tubes:}

Plain tube mild steel of diameter $22.4 \mathrm{~mm}$ (OD) with $19 \mathrm{~mm}$ (ID) and $1000 \mathrm{~mm}$ length was used prepare test tube. Pipe is cut along the axis to form two halves. Tube is cut by gas wire cutting method.

For the almond shape beads laser cutting is used. The same can be cut from CRCA (cold rolled closed annealed) sheet which is equivalent to Mild steel.These almond shape beads are weld by spot welding over the inside of each half of the tube. The staggered arrangement is done for the test tube which is found to be effective over CFD analysis. The two halves of the tube weld by using MIG (Metal Inert Gas) welding.

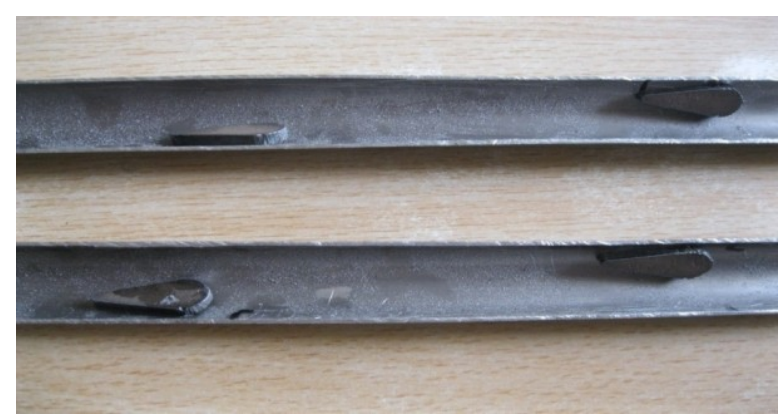

Fig.5: Photograph of Test tube section with cross dimple array 
All the problems of fluid is not possible to solve by analytically and experimentally; because of time availability and the experimentation costing.CFD is the best solution to predict the output results of difficult problem. In this experimental work CFD analysis was used to predict the results for align and staggered array of dimple tube.Staggered array gives the better heat transfer enhancement than align and plain tube. For validation of results actual experiment was carried out to get the practical outputs.

\section{Data Reduction}

Average surface temperature of test tube

$T_{s}=\frac{T_{1}+T_{2}+T_{3}+T_{4}}{4}$

$T_{b}=\frac{T_{a}+T_{5}}{2}$

Mass flow rate of air through Orifice

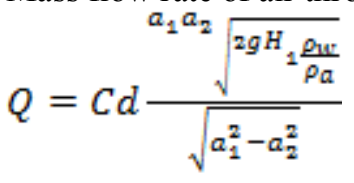

By energy balance equation heat transfer coefficient of fluid

$h=\frac{m C_{p}\left(T_{a}-T_{5}\right)}{A_{s}\left(T_{s}-T_{b}\right)}$

Nusselt numbers calculated from the experimental data for plain tube were compared with the correlation recommended by Dittus-Boelter.

Theoretical Nusselt number

$N u=0.023 \operatorname{Re}^{0.8} \mathrm{Pr}^{0.4}$

Blasius equqtion of Turblent Flow

$f=0.079 R e^{-0.25}$

LMTD

$T_{l m}=\frac{\left(T_{w}-T_{\text {in }}\right)-\left(T_{w}-T_{o}\right)}{\ln \frac{\left(T_{w}-T_{\text {in }}\right)}{\left(T_{w}-T_{o}\right)}}$

In straight pipe lengths, Pressure drop $(\mathrm{P})$ can be calculated using the Darcy Equation $\mathrm{f}=$ Darcy friction factor

friction factor $=\frac{64}{R e}$

Pressure Drop $=\frac{f l \rho v^{2}}{2 D}$

Enhancement Factor $=\frac{\text { Enthalpy change of dimple tube }}{\text { Enthalpy change of plain tubs }}$
Over all enhancement ratio $=\frac{\frac{N u}{N u_{0}}}{\left(\frac{f}{f_{0}}\right)^{1 / 3}}$

4.1 Analytical Calculations

Analytical Calculations has been carried out by using Newton Raphson's Modified Numerical Method $f(T)=U A(L M T D)-m c_{p}(\Delta T)=0$

$\left(T_{0}+h\right)=U A L M T D_{T_{0}+h}-m C_{p}\left(\left(T_{0}+h\right)-T_{i}\right)$

$f^{\prime}(t)=\frac{f\left(T_{0}+h\right)-f(T)}{h}$

$T_{\text {new }}=T_{0}-\frac{f(T)}{f^{\prime}(t)}$ 


\section{Computational Model:}

In order to obtain more physical understandings about the pressure loss and heat transfer characteristics in the pin fin-dimple channels with dimple, additional three-dimensional and steady-state conjugate numerical computations were done. The computation is performed with FLUENT Version14.5 The tube and almond shape dimple geometries employed in the computation are exactly the same as those used in the experiments. The meshing is done using GAMBIT. The numbers of the grid nodes range from about 0.5 to $1.35 \times 10^{3}$, depending on the dimple geometry and grid independence studies. Fine meshes are generated inside the dimples and around the edges of dimples to resolve key features in the vicinity of the dimple. The problem is modeled as a steady, 3dimensional heat transfer problem with a uniform wall temperature. The $k-\varepsilon$ model is employed for the calculations. This turbulence model represents the most sophisticated model available for turbulent flow calculations in FLUENT. During the computation, the tube wall is set to the constant wall temperature The uniform inlet velocity is determined by selecting the inlet Reynolds number Re15,000.The temperature of the main flow is set to be $303 \mathrm{~K}$, and the wall temperature is set to be $353 \mathrm{~K}$. Because the temperature difference is only $323 \mathrm{~K}$, and the main stream velocity is low (less than $10 \mathrm{~m} / \mathrm{s}$ ), the velocity field is assumed to be independent of temperature. The experimental and computational results were compared for the validation.

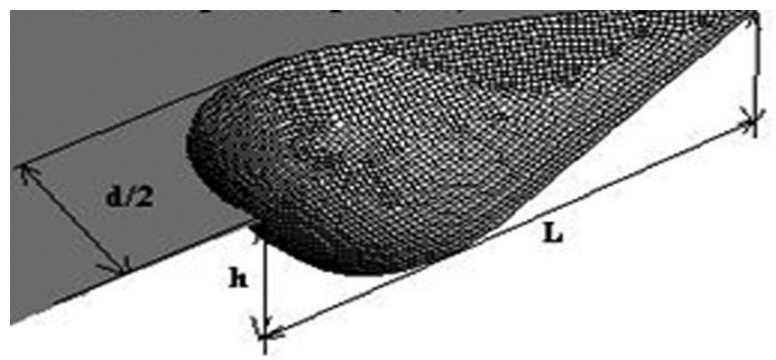

Fig.6: Geometry of dimple in computation [12]

6.1Experimental Results:

\section{Results and discussion:}

Table-1 Results of plain tube and staggered array dimple tube for varying mass flow rate.

\begin{tabular}{lllllllll}
\hline Re no & \multicolumn{2}{c}{$\mathbf{5 9 8 3 . 6 6}$} & \multicolumn{2}{c}{$\mathbf{4 2 9 9 . 7}$} & \multicolumn{6}{c}{$\mathbf{6 0 1 9 6}$} & \multicolumn{2}{c}{$\mathbf{9 4 5 9 4}$} \\
\hline Para-meter & Plain & Staggered & Plain & Staggered & Plain & Staggered & Plain & Staggered \\
$\mathbf{T}_{\mathbf{0}}$ & 62 & 69 & 59 & 65 & 56 & 63 & 54 & 59 \\
$\Delta \mathbf{H}$ & 210.475 & 256.517 & 317.905 & 383.676 & 399.02 & 5.06 .445 & 578.8064 & 699.39 \\
$\mathbf{N u}$ no & 70.321 & 95.539 & 101.727 & 134.106 & 122.508 & 171.736 & 173.027 & 223.798 \\
$\mathbf{h}$ & 89.5672 & 121.686 & 129.568 & 170.809 & 156.037 & 218.7379 & 220.3825 & 285.4 \\
$\mathbf{f}$ & 0.00819 & 0.02048 & 0.00305 & 0.00103 & 0.00316 & 0.0090 & 0.001219 & 0.0445 \\
$\Delta \mathbf{P}$ & 98.1 & 245.25 & 117.72 & 343.35 & 206.01 & 588.06 & 196.2 & 716.13 \\
\hline
\end{tabular}

Table-2 Performance factors of dimpled tube compared with plain tube

\begin{tabular}{lllll}
\hline Re no./ factor & \multicolumn{1}{c}{$\mathbf{5 9 8 3 . 6 6}$} & \multicolumn{1}{c}{$\mathbf{4 2 9 9 . 7}$} & \multicolumn{1}{c}{$\mathbf{6 0 1 9 6}$} & \multicolumn{1}{c}{$\mathbf{9 4 5 9 4}$} \\
\hline $\mathbf{\eta}_{\boldsymbol{f}}$ & 1.2187 & 1.2069 & 1.2692 & 1.20833 \\
$\Delta \mathbf{P}$ & 2.5 & 2.916 & 2.8571 & 3.65 \\
$\mathbf{\eta} \%$ & 135.14 & 131.143 & 128.812 & 116.92 \\
$\mathbf{T P F}$ & 1.00103 & 0.922 & 0.987 & 0.8400 \\
\hline
\end{tabular}

Table-3 Analytical Results for plain tube array of dimple tube

\begin{tabular}{lllll}
\hline Re no./Parameter & \multicolumn{1}{c}{$\mathbf{5 9 8 3 . 6 6}$} & $\mathbf{4 2 9 9 . 7}$ & $\mathbf{6 0 1 9 6}$ & \multicolumn{1}{c}{$\mathbf{9 4 5 9 4}$} \\
\hline $\mathbf{T}_{\mathbf{0}}$ & 59.7921 & 57.933 & 56.727 & 55.1368 \\
$\Delta \mathbf{H}$ & 195.95325 & 306.214 & 410.184 & 606.2235 \\
\hline
\end{tabular}

Table-4 Theoretical Results for Staggered array of dimple tube

\begin{tabular}{lllll}
\hline \multicolumn{1}{c}{ Reno } & \multicolumn{1}{c}{$\mathbf{5 9 8 3 . 6 6}$} & \multicolumn{1}{c}{$\mathbf{4 2 9 9 . 7}$} & $\mathbf{6 0 1 9 6}$ & \multicolumn{1}{c}{$\mathbf{9 4 5 9 4}$} \\
\hline Nu no & 67.689 & 101.859 & 133.3223 & 191.3985 \\
$\mathbf{h}$ & 86.215 & 129.7365 & 169.810 & 243.7813 \\
$\mathbf{f}$ & 0.0249 & 0.0219 & 0.02017 & 0.018019 \\
$\mathbf{\eta}_{\boldsymbol{f}}$ & 1.0390 & 1.2529 & 1.2347 & 1.153 \\
\hline
\end{tabular}

6.2 Numerical Results: 
An Experimental Study of Heat Transfer Enhancement in the Circular Channel with Almond ....

Table 5: Numerical Results for Plain Tube

\begin{tabular}{rllll}
\hline \multicolumn{1}{c}{ Re No. } & \multicolumn{1}{c}{$\mathbf{T}_{\mathbf{0}}$} & \multicolumn{1}{c}{ Nu No. } & \multicolumn{1}{c}{ h } & \multicolumn{1}{c}{ f } \\
\hline $\mathbf{2 5 7 9 9 . 8 5}$ & 56.2 & 70.09088 & 83.2017 & 0.93185 \\
$\mathbf{4 2 9 9 8 . 4 4}$ & 54.4475 & 105.4702 & 123.2886 & 0.91775 \\
$\mathbf{6 0 1 9 7 . 0 3}$ & 53.14304 & 138.0473 & 159.7974 & 0.908825 \\
$\mathbf{9 4 5 9 4 . 2 1}$ & 51.66001 & 198.17898 & 229.3921 & 0.90877 \\
\hline
\end{tabular}

Table 6: Numerical Results for almond dimple tube

\begin{tabular}{cccccccc}
\hline Re No. & $\mathbf{T}_{\mathbf{0}}$ & Nu No. & $\mathbf{h}$ & $\mathbf{f}$ & $\mathbf{h}_{\mathbf{h}} / \mathbf{h}_{\mathbf{0}}$ & $\mathbf{f} / \mathbf{f}_{\mathbf{0}}$ & TPF \\
\hline $\mathbf{2 5 7 9 9 . 8 5}$ & $\mathbf{6 9 . 9 5 8}$ & $\mathbf{9 8 . 6 6 3 5}$ & $\mathbf{1 7 6 . 8 9 4}$ & $\mathbf{1 . 4 0 7 6 5 2}$ & $\mathbf{2 . 1 2 6 1}$ & $\mathbf{1 . 5 1 0 3}$ & $\mathbf{1 . 2 2 6 8}$ \\
$\mathbf{4 2 9 9 8 . 2 5}$ & $\mathbf{6 5 . 6 0 2}$ & $\mathbf{1 3 7 . 5 9 7}$ & $\mathbf{2 2 8 . 6 4 1}$ & $\mathbf{1 . 3 0 4 6 1}$ & $\mathbf{1 . 8 5 4 5}$ & $\mathbf{1 . 4 2 1 5}$ & $\mathbf{1 . 1 6 0 2 7}$ \\
$\mathbf{6 0 1 9 5 . 3 0}$ & $\mathbf{6 3}$ & 173.389 & $\mathbf{2 7 7 . 3 8}$ & $\mathbf{1 . 2 5 6 0 1}$ & $\mathbf{1 . 7 3 5 8}$ & $\mathbf{1 . 3 8 2 0}$ & $\mathbf{1 . 1 2 7 6}$ \\
$\mathbf{9 4 5 9 4 . 2 1}$ & $\mathbf{6 0 . 5 4}$ & $\mathbf{2 4 3 . 3 6 6}$ & $\mathbf{3 8 1 . 2 7 3}$ & $\mathbf{1 . 2 2 9 0 1 6}$ & $\mathbf{1 . 6 6 2 1}$ & $\mathbf{1 . 3 5 2 3 8}$ & $\mathbf{1 . 1 1 3 6 7}$ \\
\hline
\end{tabular}

6.4 Friction Factor:

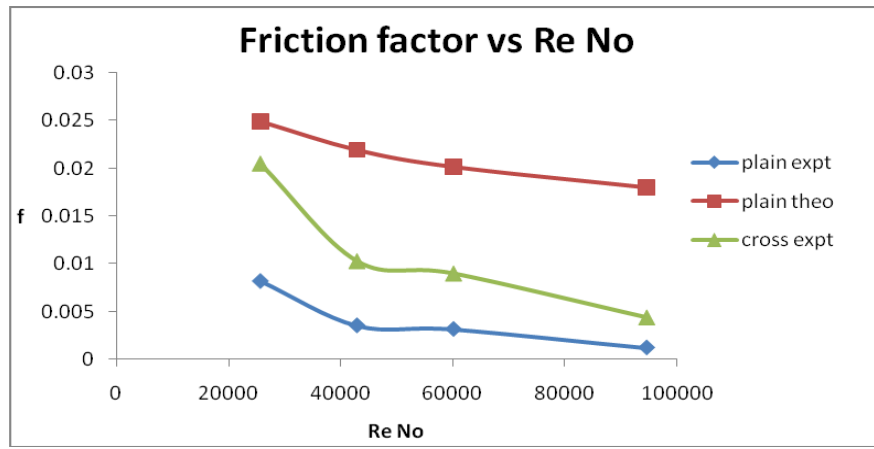

Graph 1:Friction factor Vs Reynolds No.for .Plain,Staggered array dimple tube

The friction factors of the almond staggered arrangement tube and the plain tube are presented in graph no.7.3.2 graph shows that, for the studied Reynolds number range from 25000-95000, the experimental results of the friction factors of the plain tube agree reasonably well with the empirical Blasius correlation. It can be seen that in the Reynolds number range of 25000-95000, depending on the dimple arrangement tube show different flow friction characteristics. Over the studied Reynolds number range, staggered arrangement shows the highest friction factors, which are slightly higher than almond tube with align and plain tube by about $30 \%$. Friction factor goes on decreasing as Re no increases.

\section{Discussions on the pressure loss characteristics:}

From the above discussion it can be seen that the pressure loss charactricstics are slightly higher in dimple tube but the rate of heat transfer enhancement get dominant in dimple tube as well pressure loss is also low as compare to other dimple shapes.

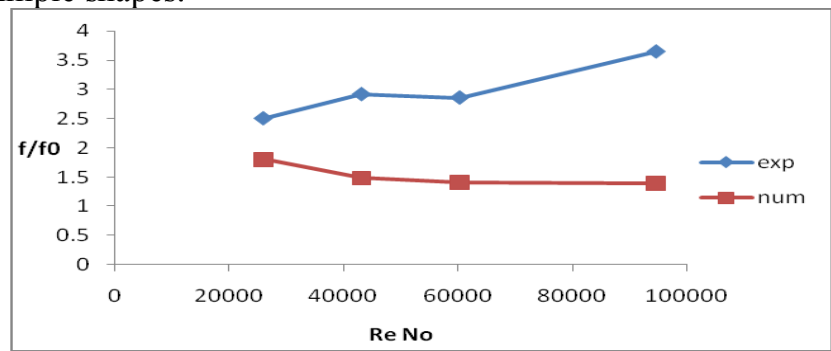

Graph2:avg. friction factor Vs Reynolds No.for .Plain,Staggered array dimple tube

6.5 Heat Transfer:

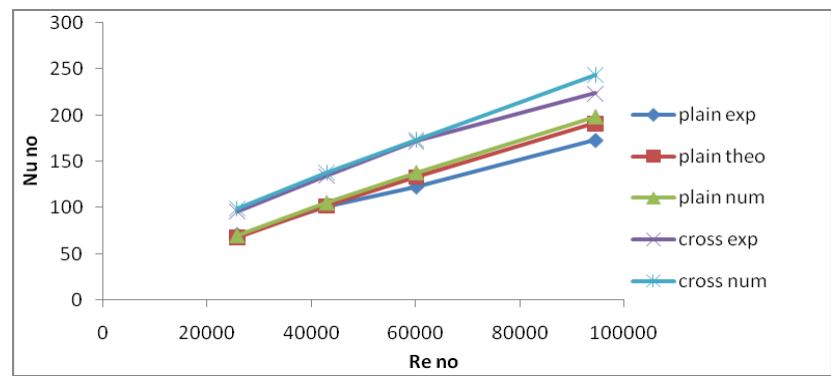

Graph.no3:Comparison of Nusselt number plain ,staggered array dimple geometries Vs Reynolds number 
Based on the experimental system shown in fig.5.4, the heat transfer characteristics for the airflow in the staggered almond attached tube have been measured respectively within the Reynolds number range of 25,000 to 95,000 . In addition, in order to validate the heat transfer measurement system in the experiments, the average Nusselt numbers of the plain tube were also measured at the same Reynolds numbers as employed in the almond tube. Graph plots comparisons of the experimental results of the average Nusselt numbers with plain tube over the Reynolds number range of 25,000 to 95,000. As is expected, within the studied Reynolds number range the Nusselt numbers of the staggered array almond tube increase with the Reynolds number. For the cross array almond Nusselt number is about 50 to $60 \%$ higher than the plain tube within the Reynolds number range of 25,000 to 95,000 .It is obvious to get the same graph plots for the heat transfer coefficient. Heat transfer enhancement can occur with the same rate in turbulent flow region due to staggered array dimples attached in circular tube.

From the above description, it can be seen that the added almond dimple arrays can further enhance the heat transfer distinctively. The main reason for the heat transfer enhancement should be that the almond shapes on tube wall surface further increase the turbulent mixing in the flow near the wall by producing multiple vortex pairs, which enhance the turbulent flow heat transfer from the wall.

6.6 The Thermal Performance:

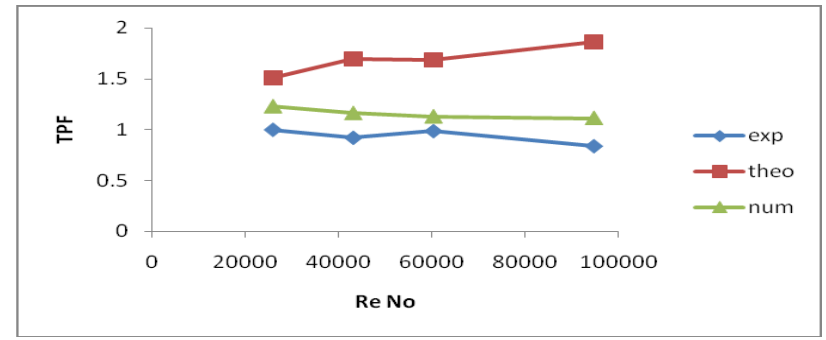

Graph.4: Thermal Performance factor Vs Re No.

Graph no4 shows the variation of thermal enhancement factor with Reynolds number. The thermal enhancement factors for the staggered array tend to decrease with increasing Reynolds number. Theoretical thermal performance is increases. With the use of almond dimples, the thermal enhancement factors are in a range between, 1 to 0.8 respectively for the Re No range 24,500 to 94,500 respectively. To properly evaluate the thermal performance of the heat exchanger, the heat transfer performance of the plain tube can only be assessed after including the penalty effects related to friction losses. The overall thermal performance parameter TPF $=$ $\left(\mathrm{Nu} / \mathrm{Nu}_{0}\right) /\left(\mathrm{f} / \mathrm{f}_{0}\right)^{1 / 3}$ represents the quantity of heat transfer per unit pumping power.

\subsection{Flow Structure and Velocity Vectors:}

The flow structure of the airflow over the dimpled surface was studied by observing the velocity vectors. The post processing capability of Fluent was used to view the velocity vectors. To analyze the flow, the vectors were observed from the spanwise and stream wise direction of the channel. This was done to investigate the presence of any secondary flows. From elementary fluid mechanics, a secondary flow is a minor flow superimposed on the primary flow and which is not predicted by simple analytical techniques. The velocity vectors showing the primary flow following the contour of the dimple for the staggered dimpled array geometry for Reynolds numbers 25795 and 94595 are shown in Figure. The velocity contour and static temperature contours, for the staggered array geometry for Reynolds number25795 and 94594 are shown in Figure

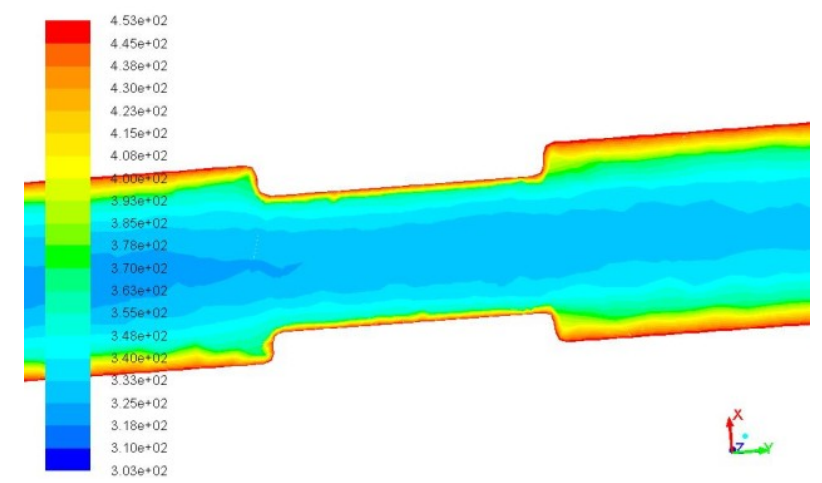

Fig.7: Static Temperature Contour Plots for $0.0065 \mathrm{~kg} / \mathrm{sec}$, Re no= 25795 


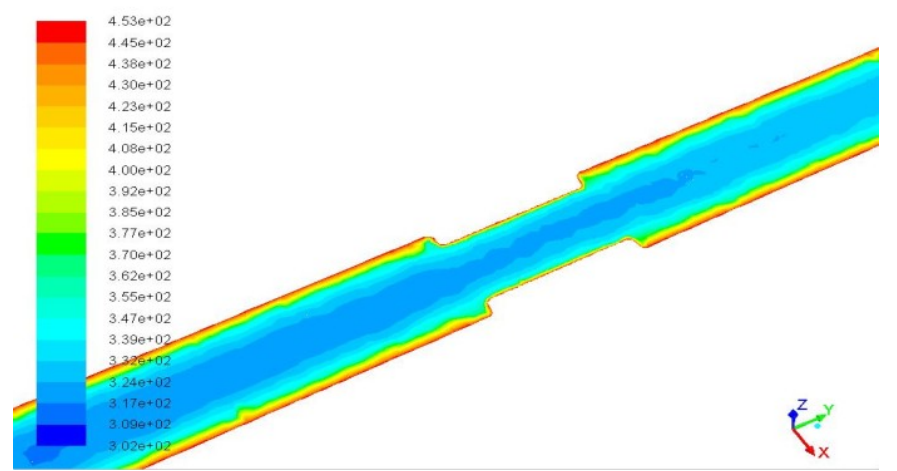

Fig.8: Static Temperature Contour Plots for $0.023997 \mathrm{~kg} / \mathrm{sec}$, Re no. $=94595$

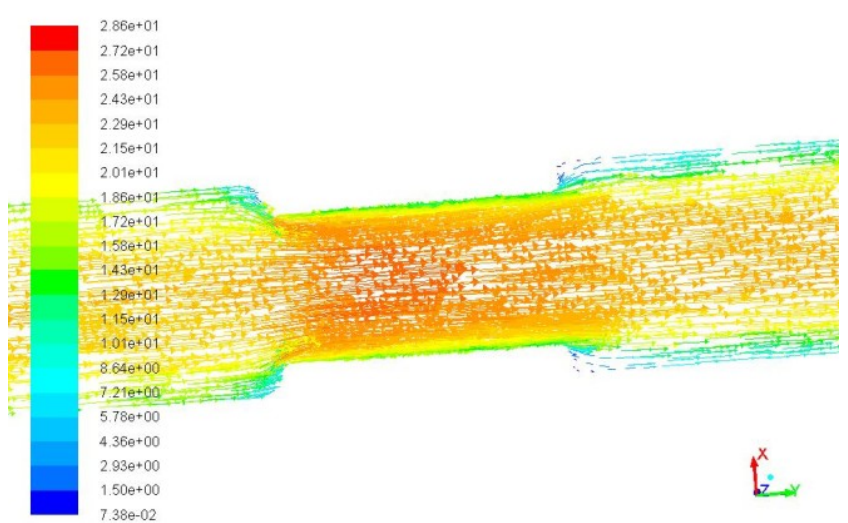

Fig.9:The Velocity Vector Plot for $0.0065 \mathrm{~kg} / \mathrm{sec}, \mathrm{Re}$ no. 25795

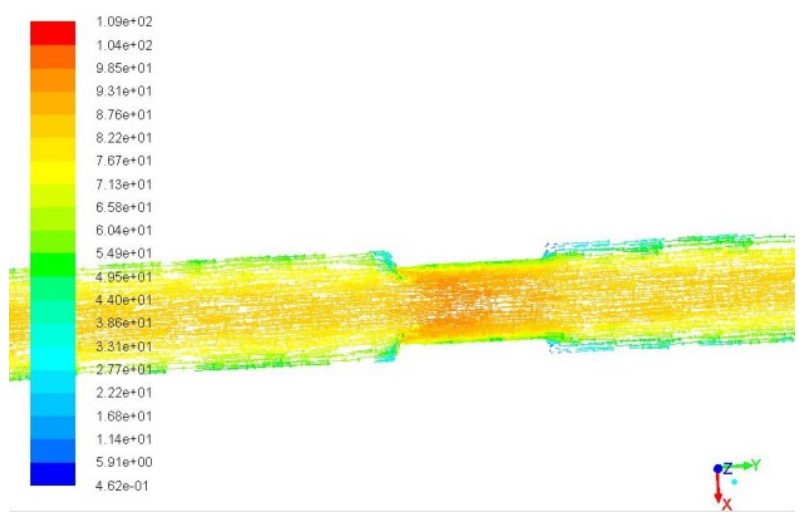

Fig.10:The Velocity contour for $0.023997 \mathrm{~kg} / \mathrm{sec}, \operatorname{Re}$ no $=94595$

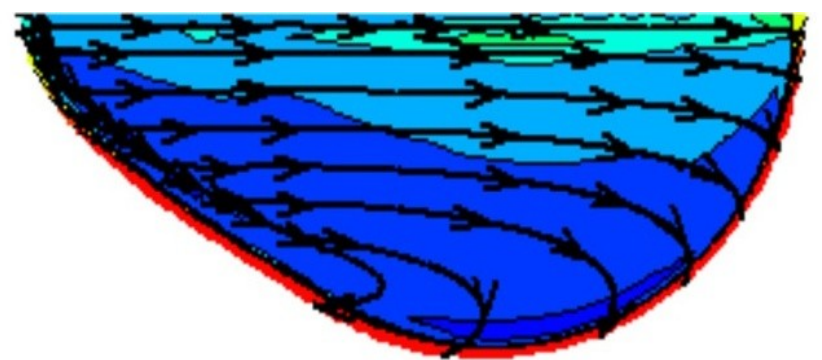

Fig.11: Stream lines on the almond dimple

The majority of the flow inside the almond shape dimple is not reversed. Only small regions of flow reversal are observed in the area adjacent to the upstream portion of the dimple surface. The incoming flow inside the almond dimple directly impinges on a large part of the dimple surface. The gentle slope of the teardrop dimple's upstream surface is the key behind the flow not experiencing a large separation. The lack of a large flow reversal and the flow impingement along the downstream edge of the dimple contribute to the greater enhancement in the heat transfer on the dimple surface. This observation indicates the most significant 
difference between the teardrop dimple and the other geometrical shapes of dimples. As the flow travels downstream and leaves the dimple, a full vortex clearly appears above the tube surface in the wake of the dimple, as shown in Fig.. Again, there are two vortices induced by the almond dimple due to the symmetry of the dimple. The vortex pair found in the almond dimple is similar to and slightly stronger than that in the circular dimple. The secondary circulations both at the upstream and downstream end of the dimple are evident from the velocity vectors seen in the span wise direction of the channel. The secondary circulations also increase in strength with increase in Reynolds number. The secondary circulations both at the upstream and downstream end of the dimple are evident from the velocity vectors seen in the span wise direction of the channel. The secondary circulations also increase in strength with increase in Reynolds number. The reattachment in case of these secondary vortexes is also evident. These secondary vortexes therefore contribute in convective heat transfer enhancement.

\section{Conclusions and Summary}

This study focused on investigating whether the use of almond dimples can enhance heat transfer characteristics for a circular tube. The staggered dimpled array geometries on the wall of a tube were tested for four different Reynolds numbers ranging from 25795 to 94594 . The dimple geometry was kept constant.

1. Nu no increases about 28 to $30 \%$ experimentally and 47 to $60 \%$ numerically in staggered array, and by analytical prediction it is almost found to be same. Variations in experimental, analytical and theoretical values are because of manufacturing and measuring errors.

2. Enhancement efficiency obtains by almond shape experimentally about 2 to $4 \%$ greater than plain tube.

3. The computations and the measurements are in good agreement with each other. The maximum error between the averaged experimental heat transfer enhancement with numerically predicted enhancement is $25 \%$.

4. The thermal performance factors were plotted for the dimpled array geometry. The thermal performance values decreased with increasing Reynolds number values. Again the thermal performance factor values for the staggered dimpled array geometry were show slightly different trend than theoretical valued. But still comparing with studied literature thermal performance is better in almond shape dimple tube.

5. The secondary vortices generated because of the dimple also help in enhancing convective heat transfer coefficient as the vortices help in mixing the hot and cold fluids. Thus, the dimple on the tube found to enhance heat transfer over a plane tube for turbulent airflows. The staggered dimpled array geometry proved to give a better thermal performance than plain tube.

6. As the friction factor goes on decreasing as Re no increases. Theoretically and experimentally for highest values of Re no it shows less deviation.

7. The gentle slope of the almond dimple's upstream surface is the key behind the flow not experiencing a large separation. The lack of a large flow reversal and the flow impingement along the downstream edge of the dimple contribute to the greater enhancement in the heat transfer on the dimple surface.

\section{Future Work}

Every research work always has a definite scope of further activity or extension of previous research work. It is ongoing process of research that the present status of the system can be changed by having certain modification, improvement, innovation, etc

The test tube material can be change such as copper, which gives better performance is compared with different material combination.

Dimples shapes variation can be done in such a way which gives maximum heat transfer en One can design a dimple tube by casting which have complete rough surface, pitch between dimples is zero. Such a tube may be give better heat transfer enhancement, but at the cost of pressure drop. One has to control the pressure loss to increase the efficiency of the heat exchanging device.

Further work part of this project will be by making efficient analysis of heat performance parameters, one can develop a correlation for the critical Reynolds number will derive for the circular tube with align and staggered almond shape dimples. Also, friction factor $\mathrm{f}$ and Nusselt number correlations will be derive with respect to the Reynolds number and geometric parameters.

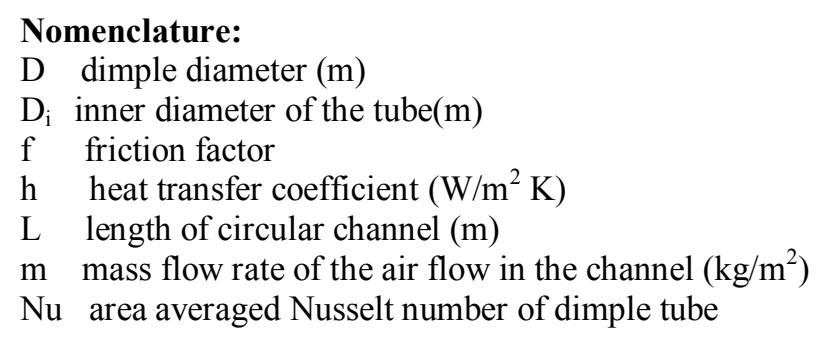


$\mathrm{Nu}_{0}$ area averaged Nusselt number of tube without dimple

Pr Prandtal number

$\triangle \mathrm{P}$ pressure drop $(\mathrm{Pa})$

$\mathrm{k}$ fluid thermal conductivity. (W/mK)

$\mathrm{Q}$ net heating power (W)

Re Reynolds number

$\mathrm{T}_{\text {in }} \quad$ inlet fluid temperature $(\mathrm{K})$

$\mathrm{T}_{\text {out }}$ outlet fluid temperature $(\mathrm{K})$

$\mathrm{T}_{\mathrm{w}}$ mean wall temperature $(\mathrm{K})$

$\Delta \mathrm{T}_{\mathrm{lm}} \log$ mean temperature difference $(\mathrm{K})$

$\mathrm{q}_{\mathrm{m}}$ mass flow rate $(\mathrm{kg} / \mathrm{sec})$

Greek symbols

$\rho$ density, $(\mathrm{kg} / \mathrm{m} 3)$

$\mu$ dynamic viscosity (Pa.s)

in inlet

Subscript

m mean

out outlet

w wall

\section{References}

[1]. Johann Turnow, Nikolai Kornev,Valery Zhdanov, Egon Hassel, "Flow structures and heat transfer on dimples in a staggered arrangement", International Journal of Heat and Fluid Flow, 2012, 35, pp. 168-175.

[2]. Yu Rao,Chaoyi Wana, Yamin Xu, "An experimental study of pressure loss and heat transfer in the pinfin-dimple channels with various dimple depths", International Journal of Heat and Mass Transfer, 2012, 55, pp. 6723-6733.

[3]. C. Bi, G.H. Tang, W.Q. Tao, "Heat transfer enhancement in mini-channel heat sinks with dimples and cylindrical grooves", Applied Thermal Engineering, 2013, 55, pp. 121-132.

[4]. S.A. Isaev, N.V. Kornev, A.I. Leontiev, E. Hassel,"Influence of the Reynolds number and the spherical dimple depth on turbulent heat transfer and hydraulic loss in a narrow channel”, International Journal of Heat and Mass Transfer, 2010, 53, pp.178-197.

[5]. Jonghyeok Lee, Kwan-Soo Lee, "Correlations and shape optimization in a channel with aligned dimples and protrusions", International Journal of Heat and Mass Transfer, 2103, 64, pp.444-451.

[6]. Somin Shin, Ki Seon Lee, Seoung Duck Park, Jae Su Kwak, " Measurement of the heat transfer coefficient in the dimpled channel:effects of dimple arrangement and channel height", Journal of Mechanical Science and Technology, 2009, 23, pp.624-630.

[7]. Yu Rao a,Yamin Xu, Chaoyi Wana, "An experimental and numerical study of flow and heat transfer in channels with pin findimple and pin fin arrays", Experimental Thermal and Fluid Science, 2012, 38, pp.237-247.

[8]. YuChen, Yong Tian Chew, Boo Cheong Khoo, " Enhancement of heat transfer in turbulent channel flow over dimpled surface", International Journal of Heat and Mass Transfer, 2012, 55, pp.8100-8121.

[9]. Nopparat Katkhaw, NatVorayos, Tanongkiat Kiatsiriroat, Yottana Khunatorn, Damorn Bunturat, AtipoangNuntaphan,"Heat transfer behavior of flat plate having $45^{\circ}$ ellipsoidal dimpled surfaces", Thermal engineering, 2013

[10]. M. Siddique, A. A. Khaled, N. I. Abdulhafiz, and A. Y. Boukhary, "Recent Advances in Heat Transfer Enhancements : A Review Report", International Journal of Chemical Engineering, 2010, id.106461.

[11]. J.E. Kim, J.H. Doo, M.Y. Ha , H.S. Yoon, C. Son,"Numerical study on characteristics of flow and heat transfer in a cooling pa ssage with protrusion-in-dimple surface", International Journal of Heat and Mass Transfer, 2012, 55, pp.7257-7267.

[12]. Sumantha Acharya," Experimental and computational study of heat/mass transfer and flow structure of four dimple array in square channel" ,Journal of Turbomachinery, 2012

[13]. M.K. Chyu, Y. Yu, H. Ding, J.P. Downs and F.O. Soechting," Concavity enhanced heat transfer in an internal cooling passage"e, International Gas Turbine \& Aeroengine Congress \& Exhibition ASME paper 97-GT-437.

[14]. Mohammad A. Elyyan," Heat Transfer Augmentation Surfaces Using Modified Dimples/ Protrusions", Ph.D, Virginia, Blacksburg, 2008.

[15]. Cengel, Y. A., Heat and Mass Transfer: A Practical Approch, $3^{\text {rd }}$ ed., Tata McGraw Hill, New York, 2010.

[16]. Versteeg, H. K. and Malalasekera, W.,An Introduction to Computational Fluid dynamics Finite Volume Method, England, 1995. 